\title{
Hyphenation of Supercritical Fluid Chromatography and NMR with In-Line Sample Concentration
}

\author{
F. H. M. van Zelst, ${ }^{\dagger,}$ S. G. J. van Meerten, ${ }^{\dagger, \dagger}$ P. J. M. van Bentum, ${ }^{\dagger}$ and A. P. M. Kentgens ${ }^{*},^{\dagger}$ \\ ${ }^{\dagger}$ Institute for Molecules and Materials (IMM), Radboud University, Nijmegen 6525 AJ, The Netherlands

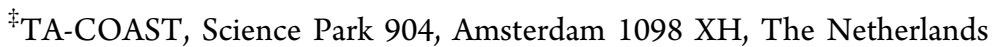

Supporting Information

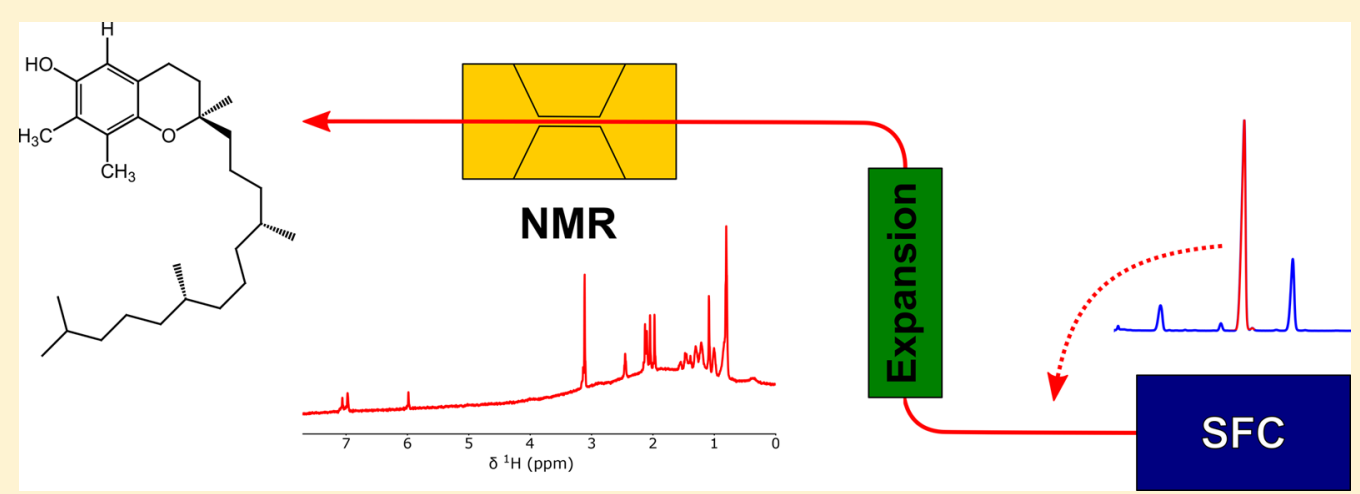

ABSTRACT: By coupling supercritical fluid chromatography (SFC) and nuclear magnetic resonance (NMR) in-line, a powerful analytical method arises that enables chemically specific analysis of a broad range of complex mixtures. However, during chromatography, the compounds are diluted in the mobile phase, in this case supercritical $\mathrm{CO}_{2}\left(\mathrm{scCO}_{2}\right)$, often resulting in concentrations that are too low to be detected by NMR spectroscopy or at least requiring excessive signal averaging. We present a hyphenated SFC-NMR setup with an integrated approach for concentrating samples in-line, which are diluted in $\mathrm{scCO}_{2}$ during chromatography. This in-line concentration is achieved by controlled in-line expansion of the $\mathrm{scCO}_{2}$. As a proof of concept four isomers of vitamin $\mathrm{E}$ (tocopherol) were isolated by SFC, concentrated in-line by expanding $\mathrm{CO}_{2}$ from 120 to 50 bar, and finally shuttled to the NMR spectrometer fitted with a dedicated probehead for spectroscopic characterization of microfluidic samples. The abundant isomers were readily detected, supporting the viability of SFC-NMR as a powerful analytical tool.

\begin{abstract}
A nalyzing complex mixtures, which are often only available

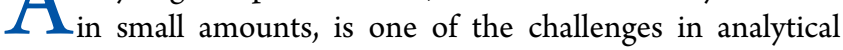
chemistry. To analyze these mixtures, it is necessary to separate the molecular components in the mixture prior to spectroscopic characterization. Common hyphenated chromatographic techniques couple the separation by liquid chromatography (LC) or gas chromatography (GC) to detection by a photodiode array (PDA) detector or mass spectrometer (MS). ${ }^{1}$ Although PDA or MS detection is often used, the structural information that can be gained from these detection techniques is limited compared to nuclear magnetic resonance spectroscopy (NMR). ${ }^{2}$ The main advantages of using NMR as a detector is that it is quantitative and provides chemical resolution. It is therefore the preferred technique for obtaining detailed molecular structures without prior knowledge of the molecule. Many examples of separation techniques hyphenated with NMR have appeared over the years, ${ }^{3,4}$ such as capillary HPLC-NMR ${ }^{5,6}$ having the advantage of low solvent volumes, GC-NMR ${ }^{7}$ for volatile compounds, and many examples of (HP)LC-NMR, ${ }^{8}$ often with parallel MS detection. ${ }^{9}$
\end{abstract}

As an alternative to LC or GC, supercritical fluid chromatography (SFC) can be used for chromatographic separation. In SFC, supercritical carbon dioxide $\left(\mathrm{scCO}_{2}\right)$ is used as the mobile phase. Due to the higher diffusivity and lower viscosity of supercritical fluids, ten times higher flow rates are achieved in SFC than in normal phase LC. Another advantage of using SFC instead of LC when hyphenated with NMR is the mobile phase. In LC-NMR solvent suppression techniques are necessary to reduce signal coming from the mobile phase. In SFC-NMR however, the mobile phase, $\mathrm{scCO}_{2}$, does not give a ${ }^{1} \mathrm{H} \mathrm{NMR}$ signal, so "cleaner" spectra are obtained. Furthermore, $\mathrm{scCO}_{2}$ is a "green" and low-cost solvent, unlike the organic solvents normally employed in LC. By adding small amounts of other solvents to the $\mathrm{scCO}_{2}$, known as modifiers or cosolvents, the polarity of the mobile phase can be optimized so that a broad range of compounds can be separated. A modifier gradient in $\mathrm{CO}_{2}$ is often used to

Received: May 27, 2018

Accepted: August 6, 2018

Published: August 6, 2018 
speed up the chromatography, eluting the more polar compounds with an increasingly polar mobile phase. ${ }^{4}$ The downside of using these modifiers is that their signals will show up in the NMR spectra of the sample of interest. The amount of modifier is however much smaller (typically a few percent) than in LC. Their signal can be suppressed by switching to deuterated solvents after chromatography, which will be described in more detail later in this paper. If SFC and NMR can be coupled, then a powerful tool for analyzing a broad range of complex mixtures becomes available.

Although SFC-NMR has been reported earlier as an analysis technique ${ }^{10-12}$ the limitations in the sensitivity of NMR make the technique less popular, especially for nanoscale sample volumes. To compensate for the low sensitivity, large amounts of sample are needed to obtain a good signal. However, this leads to overloading of the chromatographic column, thus reducing the chromatographic resolution. ${ }^{13}$ To solve this issue, the sensitivity of NMR for small sample volumes must be improved and the sample should be concentrated before analysis in the NMR spectrometer.

Several innovations have been made to enhance the sensitivity in NMR for small sample volumes. By miniaturizing the detection coil, smaller sample volumes can be measured. Research has been focused on developing tightly wound solenoid coils and planar helical microcoils. ${ }^{14-17}$ An alternative approach was the development of planar microslot waveguide probes. ${ }^{18}$ The challenge in miniaturization is to get the highest sensitivity while maintaining an uncompromised high resolution. ${ }^{19}$ Over the past few years our group has introduced onchip stripline detectors for high-resolution NMR of masslimited samples. ${ }^{20}$ The conventional coil geometry used for detection has been replaced by flat stripline RF inductors. The flat geometry of the stripline can be used for in-flow detection, by passing a capillary over the stripline. Stripline probes can achieve a high resolution and a single scan sensitivity of 0.1 nanomole ${ }^{1} \mathrm{H}$ spins per square-root $\mathrm{Hz}$ receiver bandwidth and of a few nanomoles for ${ }^{13} \mathrm{C}$ for sample volumes from $100 \mathrm{~nL}$ to $1 \mathrm{~mL}^{20,21}$

To improve sensitivity further, the sample can be concentrated before analysis. In the work by Tayler et al. this was done by evaporating the sample off-line before detecting it with NMR. ${ }^{22}$ The authors first separated a mixture of four isomers of vitamin E (tocopherol) by SFC. Each isomer (Figure S1b of the Supporting Information, SI) was then collected separately in a vial 10 times, the excess solvent was removed and each isomer was redissolved in $0.5 \mu \mathrm{L}$ methanol$\mathrm{d}_{4}$. This small amount of sample was then inserted into a capillary, and NMR spectra were acquired during $25 \mathrm{~min}$ for each isomer, averaging over 500 scans with a relaxation delay of 2 s. Implementing an in-line concentration step between chromatography and spectroscopy would greatly reduce the analysis time, and make automated and more quantitative analysis possible by reducing losses in the sample collection and preparation steps. In this study, the possibility to concentrate samples, which are diluted in $\mathrm{scCO}_{2}$ during chromatography, is investigated.

In the high density liquid regime of $\mathrm{CO}_{2}$, above the critical temperature and pressure of $31.9^{\circ} \mathrm{C}$ and 73 bar respectively, ${ }^{13}$ molecules can be dissolved into $\mathrm{CO}_{2}$. By changing the pressure and or temperature of a supercritical fluid, its density changes and thereby its solvating ability. When supercritical $\mathrm{CO}_{2}$ expands and becomes a gas, the density decreases with decreasing pressure and the sample will not stay dissolved.
This principle is used for implementing an in-line concentration step to convert the off-line SFC-NMR setup into an inline analysis technique.

\section{EXPERIMENTAL SECTION}

Set-Up. In our initial SFC-NMR setup, in which SFC was coupled directly to the NMR probe through a flow control system, the pressure of the SFC system and the pressure of the tubing going to the NMR probe was controlled by the same backpressure regulator at 120 bar. However, the concentration of the sample in the plug was too low to detect by NMR spectroscopy within a reasonable amount of time. Therefore, the system was extended with an in-line concentrator (indicated in green in Figure 1a). In this setup, the tubing

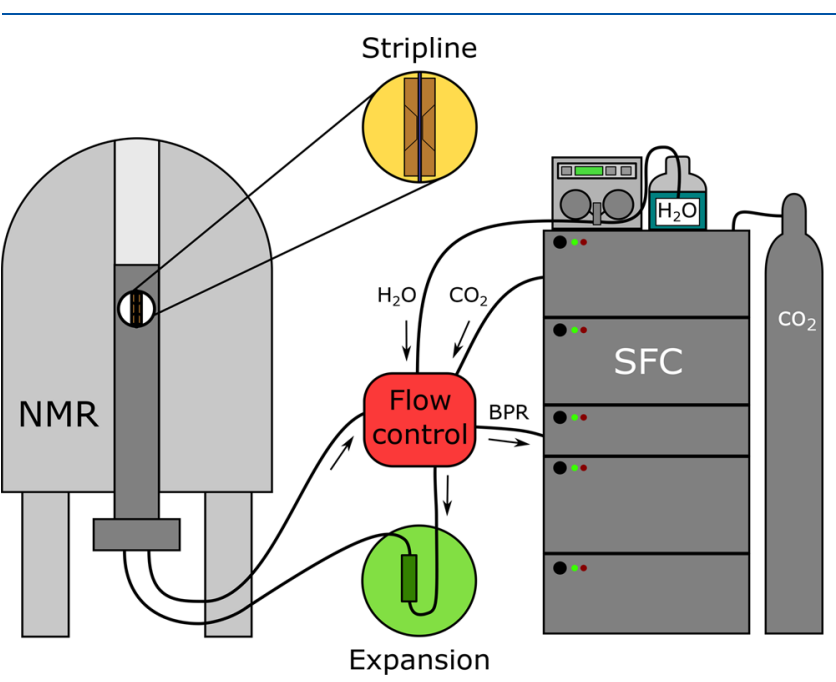

(a)

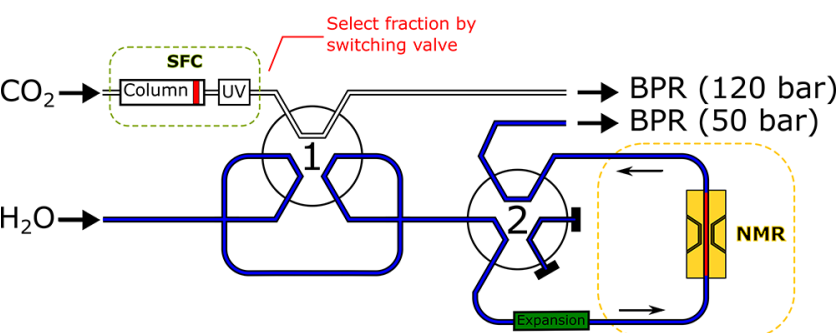

(b)

Figure 1. (a) Schematic representation of the SFC-NMR system. A broad expansion tube (green) is added before the NMR spectrometer for concentrating the sample. (b) The flow control system: After SFC, a fraction of interest can be selected from the UV chromatogram using valve 1 , flowing into a $100 \mu \mathrm{L}$ sample loop. The water flow pushes this plug toward the center of the NMR stripline chip at a rate of $0.1 \mathrm{~mL} / \mathrm{min}$. By switching valve 2, the flow can be stopped to acquire multiple scans.

going to the NMR probe is connected to a different manual backpressure regulator with an operating range between 20 and 103 bar (Vici Jour). This backpressure regulator allows us to lower the pressure of a sample fraction which was selected after SFC. Lowering the pressure leads to expansion of the plug in the water flow. An extra stainless steel tube, with an inner diameter of $2.159 \mathrm{~mm}$ and length of $\sim 20 \mathrm{~cm}$, is added to the system before the NMR probehead to allow for separation of 
the phases $\left(\mathrm{scCO}_{2}\right.$, gas phase $\mathrm{CO}_{2}$ and sample), as shown in Figure 1. The PEEK tubing going from the expansion tube to the bottom of the NMR probe has an inner diameter of 0.254 $\mathrm{mm}$. All other PEEK tubing is $0.508 \mathrm{~mm}$ wide. The fused silica capillary (Polymicro Technologies) in the stripline probe has an inner diameter of $250 \mu \mathrm{m}$ and outer diamer of $360 \mu \mathrm{m}$. To select the desired fraction coming from the SFC instrument and to stop the flow in the NMR probehead, two 6-port binary-position valves (Vici Valco, Inc.) can be switched, namely valve 1 and valve 2 in Figure $1 \mathrm{~b}$, respectively.

The flow control system of the SFC-NMR setup can be seen in detail in Figure 1b. In the SFC machine, the sample is injected into a $\mathrm{CO}_{2}$ flow and loaded onto the column for separation at high pressure (120 bar in this case). This separation is followed by UV detection, after which the sample goes through the backpressure regulator to the waste. Once a sample of interest passes the UV detector, valve 1 can be switched. A $100 \mu \mathrm{L}$ loop is then filled with the sample, $\mathrm{scCO}_{2}$ and possibly a cosolvent. Once the loop is filled, the valve switches back, thus inserting the sample plug into a flow of an incompressible and immiscible medium, water in our case. This separate water flow line, leading to the NMR probe, is kept at a lower pressure to expand the $\mathrm{scCO}_{2}$ plug. After passing a wider tube, the sample is concentrated at the trailing edge of the plug (as will be discussed in the Results section) and flows at a rate of $0.1 \mathrm{~mL} / \mathrm{min}$ toward the center of the stripline for detection. This sample can be detected by NMR in flow, or the flow can be stopped by switching the second valve. In stop-flow mode, more scans can be acquired to enhance signal intensities. The system remains under pressure when the flow is stopped. After the NMR experiment, the sample will flow through the backpressure regulator to the waste.

Instruments. In the SFC-NMR setup, we coupled a Waters Acquity $\mathrm{UPC}^{2}$ instrument to a Varian VNMRS spectrometer at $600 \mathrm{MHz}$ Larmor frequency $(14.1 \mathrm{~T})$. A home-built stripline probe was used, ${ }^{20}$ with a $300 \mu \mathrm{m}$ wide and $4 \mathrm{~mm}$ long chip, optimized for detecting an active volume of $150 \mathrm{~nL}$. In-flow experiments were performed with an acquisition time of $0.5 \mathrm{~s}$ and a relaxation delay of $0.2 \mathrm{~s}$ (in total $0.7 \mathrm{~s}$ per spectrum), while stop-flow experiments were recorded with an acquisition time of $1 \mathrm{~s}$ and a relaxation delay of $5 \mathrm{~s}$ (for the samples separated in methanol $/ \mathrm{CO}_{2}$ ) or $10 \mathrm{~s}$ (for the sample separated in toluene $\left./ \mathrm{CO}_{2}\right)$. All spectra were recorded with a receiver bandwidth of $10 \mathrm{kHz}$. The spectra are referenced to the $\mathrm{CH}_{3}$ peak of toluene, which was set to $2.1 \mathrm{ppm}$.

SFC Chromatography. A mixture of four vitamin E (tocopherol) isomers (Sigma-Aldrich, CAS 1406-66-2, not less than $80 \% \beta, \gamma$ and $\delta$ isomers) was diluted in toluene (Fisher Scientific) at $100 \mathrm{mg} / \mathrm{mL}(0.24 \mathrm{M})$. Two $\mu \mathrm{L}$ of this sample (0.2 mg tocopherol) was injected onto a $100 \mathrm{~mm} \times 1.7$ $\mu \mathrm{m}$ packed BEH SFC column (Waters) at a backpressure of $120 \mathrm{bar}$, a temperature of $50{ }^{\circ} \mathrm{C}$ and a flow rate of $1.5 \mathrm{~mL} / \mathrm{min}$. For the separation in toluene in $\mathrm{CO}_{2}$ (Linde Gas Benelux, food grade), a linear solvent gradient of 20 to $30 \%$ toluene $/ \mathrm{CO}_{2}$ in 4 min was used. For the separation in methanol $/ \mathrm{CO}_{2}$, a linear solvent gradient of 2 to $5 \%$ methanol (BioSolve, SFC grade) in $\mathrm{CO}_{2}$ in 3 min was used.

\section{RESULTS AND DISCUSSION}

Expansion of Toluene in Supercritical $\mathrm{CO}_{2}$. Separation of the four isomers of tocopherol, differing in the methylation pattern of the aromatic ring, has been achieved before by SFC. ${ }^{22}$ The same tocopherol mixture as used by Tayler et al. has been used in this research consisting approximately of $9.7 \%$ $\alpha$-, $1.1 \% \beta$-, $66.7 \% \gamma$-, and $22.5 \% \delta$-tocopherol, determined by integration of their separate peaks in the UV chromatogram (SI S1). A good separation can be achieved within $2.5 \mathrm{~min}$ using a gradient of methanol in $\mathrm{CO}_{2}$, but the $\beta$ - and $\gamma$-isomers slightly coelute. The SFC column can be loaded with up to 0.2 $\mathrm{mg}$ of tocopherol mixture during each injection. Selecting 100 $\mu \mathrm{L}$ of the peak containing the highest abundant isomer, $\gamma$ tocopherol ( $66.7 \%$ of the mixture, $0.13 \mathrm{mg}$ ), and shuttling this compound directly to the NMR probe, without in-line concentration, would result in an isomer concentration of 0.2 $\mu \mathrm{g}(3.2 \mathrm{mM})$ in the $150 \mathrm{~nL}$ NMR detection volume of the stripline. As mentioned in the introduction, the limit of detection (LOD) for the stripline chip used in these experiments is $0.1 \mathrm{nmole}{ }^{1} \mathrm{H}$ spins $/ \sqrt{\mathrm{Hz}} \cdot{ }^{20,22}$ This detection limit is defined as the number of nuclear spins of a specific molecular site needed for a signal-to-noise ratio of 1 in the FID, for a single scan experiment. When more scans are acquired and averaged, a better signal-to-noise ratio is achieved, scaling with the square root of the number of scans. For a single scan experiment, with a receiver bandwidth of $10 \mathrm{kHz}$, the concentration LOD is $67 \mathrm{mM}$ in the $150 \mathrm{~nL}$ detection volume of this stripline for a single resonance in the spectrum. However, if 500 scans are acquired, then the concentration LOD would be $3 \mathrm{mM}$ for a signal-to-noise ratio of 1 in the FID for a single resonance. The concentration of $\gamma$ tocopherol that comes from the SFC with maximum loading of the column $(3.2 \mathrm{mM})$ is therefore the bare minimum required for NMR detection within reasonable time $(3 \mathrm{mM}$ in 500 scans, taking approximately an hour of experiment time). The other, less abundant isomers will not be visible within the same experiment time when SFC and NMR are coupled directly. However, if an in-line concentration step is implemented between SFC and NMR, then these isomers can also be detected. On the basis of the detection limits and sample amounts stated above, a concentration step of approximately 57 times would be needed for the isomer with the lowest abundance (the $\beta$-isomer), and of approximately 6.5 times for the second lowest abundant isomer (the $\alpha$-isomer) to obtain the detectable concentration of $3 \mathrm{mM}$.

The calculations above set the minimum goal for the in-line concentration step. The experiments in the following sections will show if this goal can be achieved.

The in-line concentration step can be achieved by expanding the $\mathrm{scCO}_{2}$ in which the sample of interest is dissolved after chromatography, as explained below. The solubility of compounds in $\mathrm{CO}_{2}$ depends on its density. By reducing the pressure, thus expanding the $\mathrm{scCO}_{2}$ into a gas, its solvating power is expected to decrease. Therefore, if the pressure drops, then the sample and, if present, the cosolvent, will no longer dissolve in the $\mathrm{CO}_{2}$, thereby achieving an in-line separation of the sample from the mobile phase. To test this hypothesis, NMR experiments were performed at different pressures to monitor the expansion, of which the results at a final pressure of 100 bar respectively 50 bar are shown in Figure 2. A mixture of toluene in $\mathrm{scCO}_{2}$ is used as a model compound. The experiment was designed to test if separating $100 \mu \mathrm{L}$ of $8 \%$ toluene dissolved in $\mathrm{scCO}_{2}$ at $120 \mathrm{bar}$ is possible by expanding the $\mathrm{CO}_{2}$ in a broad tube. In Figure 2, an array of ${ }^{1} \mathrm{H}$ NMR spectra is shown, zoomed in on the $\mathrm{CH}_{3}$ peak of toluene (2.1 $\mathrm{ppm}$ ). The spectra were recorded every $0.7 \mathrm{~s}$ in flow.

When the toluene/ $\mathrm{CO}_{2}$ plug is expanded from 120 to 100 bar (Figure 2a), toluene is spread over the whole $\mathrm{CO}_{2}$ plug, 


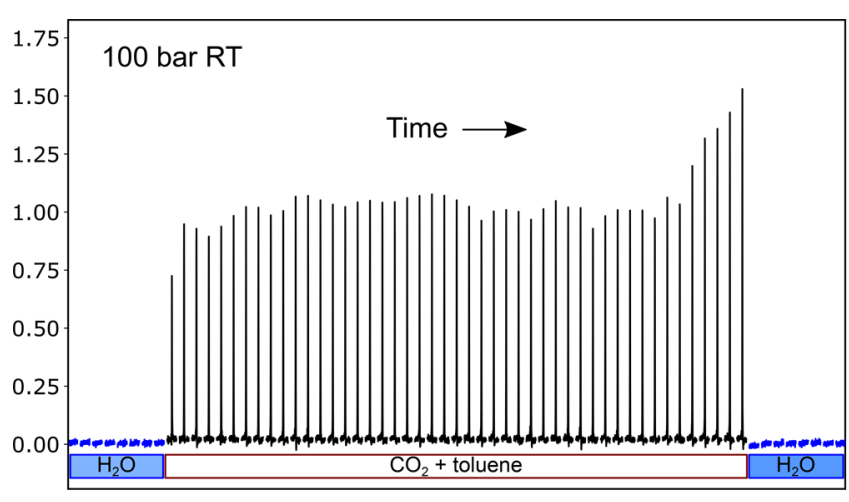

Array of ${ }^{1} \mathrm{H}$ NMR spectra

(a)

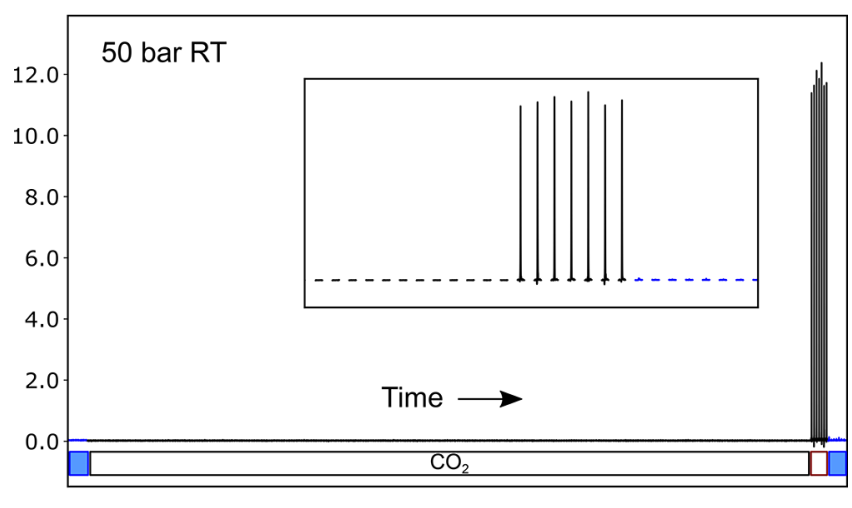

Array of ${ }^{1} \mathrm{H}$ NMR spectra

(b)

Figure 2. Array of ${ }^{1} \mathrm{H}$ NMR spectra, zoomed in on the $\mathrm{CH}_{3}$-peak of toluene, recorded during the expansion of a plug of $8 \%$ toluene in 100 $\mu \mathrm{L} \mathrm{scCO}$, in a water flow of $0.1 \mathrm{~mL} / \mathrm{min}$. The spectra in both subfigures are scaled so that direct comparison of peak intensity between the figures is possible. The spectra containing water are indicated in blue, the other peaks originate from toluene (red rectangle). The plug in (a) was expanded from 120 to 100 bar, and in (b) the plug was expanded to 50 bar. The latter gives the best results for concentrating toluene in $\mathrm{scCO}_{2}$.

indicating that toluene is still dissolved in $\mathrm{CO}_{2}$. If the pressure is decreased to 50 bar (Figure 2b), then toluene is observed only at the trailing edge of the plug in a much smaller volume and therefore in a higher concentration (based on the peak intensities, a factor 10 higher). This indicates that during the expansion to $50 \mathrm{bar}$, toluene no longer dissolves in $\mathrm{CO}_{2}$. In this way, a separation of toluene and $\mathrm{CO}_{2}$ is achieved. Once the water flow passes, at the trailing edge of the plug, the toluene is pushed forward by the water flow, since toluene is insoluble in water. This results in a concentrated toluene front at the trailing edge of the $\mathrm{CO}_{2}$ before the water, as shown in Figure 2b. A small amount of $\mathrm{CO}_{2}$ might still be dissolved in this toluene front, but this cannot be observed with ${ }^{1} \mathrm{H}$ NMR. In the next section, the expansion mechanism will be investigated in more detail. Due to the expansion into a gas, which has a lower density, the $\mathrm{CO}_{2}$ plug at 50 bar has a larger volume than the plug at 100 bar, even though before expansion the starting volume of $\mathrm{scCO}_{2}$ was the same for both experiments. This is why more NMR spectra were recorded in the second experiment than in the first, to follow the entire, expanded plug.

Expansion to even lower pressures should result in a higher concentration. However, when the plug was expanded to 40 bar, the toluene front was no longer intact and toluene droplets were observed in the water flow after the $\mathrm{CO}_{2}$. We concluded that expanding $\mathrm{CO}_{2}$ must be controlled and cannot be too sudden.

An increase in temperature leads to an even lower $\mathrm{CO}_{2}$ density, so more expansion of the gas and therefore better separation of the $\mathrm{CO}_{2}$ from the toluene and thus to an even higher concentration of toluene. Going from room temperature to $90{ }^{\circ} \mathrm{C}$ results in approximately 2.5 times higher peaks in the spectra, as shown in SI S2. In combination with the concentration of $\sim 10$ times that was already achieved by lowering the pressure to 50 bar (Figure 2), a total concentration factor of 20 to 25 can be achieved by lowering the pressure and working at elevated temperatures. However, since these results at room temperature were satisfactory and the experiment is easier to perform, we decided to perform the following experiments at room temperature. Moreover, in general terms, high temperatures might not always be desirable as some compounds might be thermally unstable at $90{ }^{\circ} \mathrm{C}$.

Expansion Mechanism. In the previous section, it was described how the separation of toluene and $\mathrm{CO}_{2}$ can be achieved by expanding the plug. To research the expansion mechanism of the plug, the forward pressure on the water pump was measured over time (Figure 3 ). The pressure of the manual, low-pressure backpressure regulator at the end of the flow line was kept constant during these experiments.

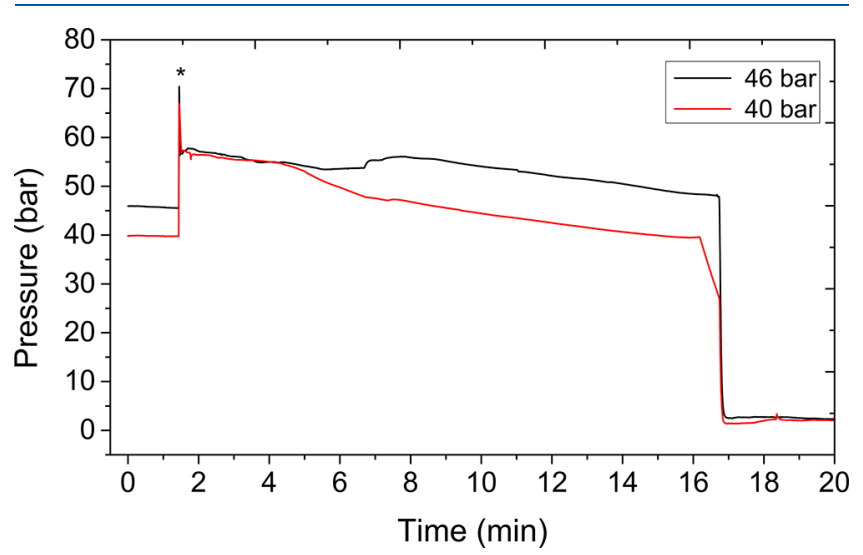

Figure 3. Forward pressure on water pump recorded during the expansion experiment. The backpressure regulator was originally set to $40 \mathrm{bar}$ (red) and $46 \mathrm{bar}$ (black). The first peak (*) originates from selecting a sample fraction at 120 bar and injecting it into the flow at lower pressure, 40 or 46 bar.

The pressure of the water flow increases after switching in the $\mathrm{scCO}_{2}$ plug (indicated by a star in Figure 3). This was expected as the plug coming from the SFC column has a pressure of 120 bar, which is much higher than the 40 or 46 bar of the water flow. An unexpected result was, however, that after switching the plug into the flow, the pressure always dropped to $57 \mathrm{bar}$, stayed at this level for about $1 \mathrm{~min}$ and then slowly decreased to the set value of the backpressure regulator (40 or 46 bar). A pressure of 57 bar which is observed is actually equal to the vapor pressure of $\mathrm{CO}_{2}$ at room temperature. This leads to the conclusion that after switching 
the valve, two phases of $\mathrm{CO}_{2}$ are present simultaneously, a liquid and a gas phase. The decrease in pressure indicates that the liquid phase slowly evaporates into the gas phase until it is fully expanded. Since the gas phase has a lower solubility than the liquid phase, the assumption is made that the sample and cosolvent present in the $\mathrm{CO}_{2}$ will stay in the liquid $\mathrm{CO}_{2}$ phase, leading to an increased sample concentration.

The expansion starts directly when injecting the plug into the PEEK tubing at the beginning of the water flow-line. This PEEK tubing was made long enough for the plug to fully expand before reaching the stripline detector. The wide stainless steel expansion tube, that is placed before the stripline detector, plays an essential role. This tubing needs to be put in a vertical position in order for the liquid phase containing the sample to be collected at the trailing edge (bottom) of the plug. When the wide stainless steel tube is put in a horizontal position, several droplets are observed before the liquid sample front at the trailing edge of the plug. It is assumed that gravity helps in separating the gaseous and liquid phase, so that all the liquid containing the sample is collected at the bottom of the expansion tube and the gas can flow to the top. Therefore, the liquid phase is always observed at the trailing edge of the plug.

SFC-NMR of Tocopherol Isomers in Toluene/ $\mathrm{CO}_{2}$. Now that we have shown that separating toluene when mixed with $\mathrm{scCO}_{2}$ is possible, the next step is to show that a sample, which was separated by SFC, can be concentrated in the same way. This sample is present in a much lower concentration than the toluene tested in the previous sections. The experimental conditions where similar to the model described in the first results section, where toluene is separated from $\mathrm{scCO}_{2}$ by expanding. Instead of only having a mixture of toluene and $\mathrm{CO}_{2}$, now a small amount of sample is added, i.e., toluene is now our cosolvent. For this experiment $0.2 \mathrm{mg}$ of a mixture of four tocopherol isomers was separated in toluene/ $\mathrm{CO}_{2} \cdot \gamma$-tocopherol was selected and shuttled into a $100 \mu \mathrm{L}$ sample loop, together with the mobile phase consisting of $25 \%$ toluene in $\mathrm{CO}_{2}$. This plug, consisting of $25 \mu \mathrm{L}$ toluene, a small amount of $\gamma$-tocopherol (less than $0.13 \mathrm{mg}$ ) and $75 \mu \mathrm{L} \mathrm{scCO}_{2}$, was put into a water flow at 50 bar at room temperature to expand the plug. When the plug reached the center of the active detection volume in the spectrometer, the flow was stopped and 500 scans were recorded in $90 \mathrm{~min}$ (Figure 4). In the case of a perfect separation of $\mathrm{CO}_{2}$ and toluene, $0.8 \mu \mathrm{g} \gamma$ tocopherol should be present in the active detection volume of the stripline $(0.13 \mathrm{mg}$ in $25 \mu \mathrm{L}$ toluene, of which $150 \mathrm{~nL}$ can be detected). The real amount is smaller since there is still some $\mathrm{CO}_{2}$ mixed in with the toluene at the trailing edge of the plug, so the sample is therefore diluted in a larger volume than the $25 \mu \mathrm{L}$ pure toluene.

One of the peaks needed to distinguish between the isomers, i.e., the aryl-H peak, indicated in red in the figure, is clearly visible after acquiring 500 scans. The aryl- $\mathrm{CH}_{3}$ peaks overlap with the broad toluene peak at $2.1 \mathrm{ppm}$. On the basis of this spectrum it can be concluded that either the $\gamma$ - or $\beta$-tocopherol isomer was isolated. To further distinguish between the two isomers the aryl- $\mathrm{CH}_{3}$ peaks must be visible. Concentrating the sample in $25 \mu \mathrm{L}$ toluene was however successful, since the sample can be detected by NMR. A broad signal from 1 to 3 ppm can be observed in the baseline of the spectrum, which originates from the coating of the fused-silica capillary. A small water contamination is present as well, but does not interfere with the tocopherol region in the spectrum. It can therefore be concluded that concentration by expansion after SFC is

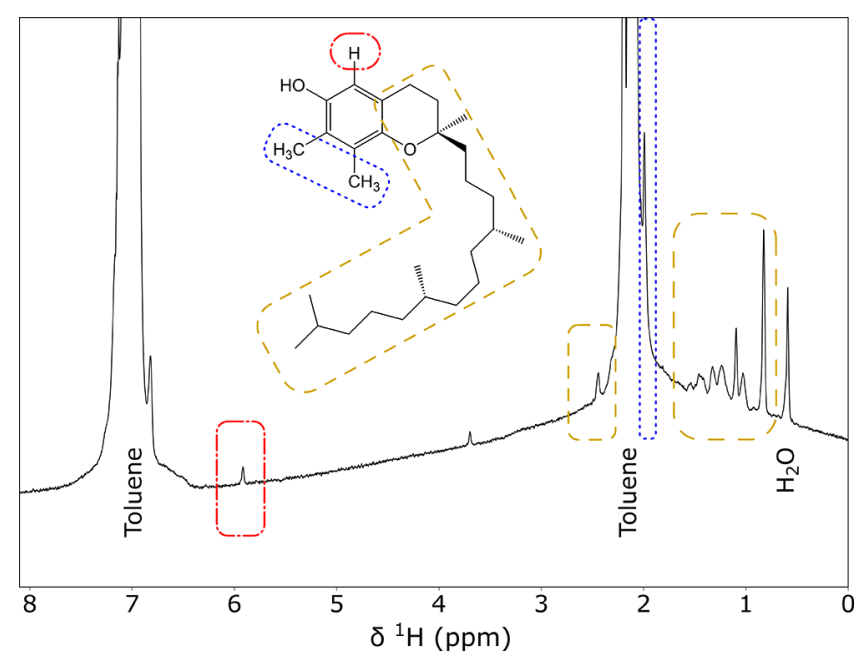

Figure 4. SFC $-{ }^{1} \mathrm{H}$ NMR spectrum of $\gamma$-tocopherol in toluene, obtained after selecting the correct isomer in SFC with 25\% toluene/ $\mathrm{CO}_{2}$ and expanding the plug to $50 \mathrm{bar}$ at room temperature, in order to increase the concentration. The flow was stopped in the NMR spectrometer and 500 scans were averaged to obtain this spectrum, taking $90 \mathrm{~min}$. The structure of $\gamma$-tocopherol and the corresponding peaks are indicated in the figure. The aryl-H peak (red), which is needed to distinguish between the isomers of tocopherol, is visible. The aryl- $\mathrm{CH}_{3}$ (blue) peaks fall below the broad toluene peak.

successful and sufficient for in-line detection by NMR. In principle, this concentration method is applicable to any nonpolar sample separated in SFC with $\mathrm{CO}_{2}$ and a nonpolar cosolvent as the mobile phase.

SFC-NMR of Tocopherol Isomers in Methanol $\mathrm{CO}_{2}$. Although we successfully separated the tocopherol isomers by SFC using toluene as a cosolvent, separation of tocopherols and many other samples, is usually achieved with methanol as cosolvent. The chromatography of tocopherol in methanol/ $\mathrm{CO}_{2}$ was optimized using a gradient of $2-5 \%$ methanol $/ \mathrm{CO}_{2}$ in $3 \mathrm{~min}$. However, when performing the SFC-NMR experiment described in the previous section with methanol instead of toluene as cosolvent, no sample or methanol were observed in the plug. This is due to the fact that methanol is polar and dissolves into the water instead of making a front on top of the water as toluene does. The sample is therefore not concentrated, but diluted further into the water flow. One possible solution to this problem is to replace the water by a nonpolar "transportation medium". This will be researched in the future. The other option is an in-line solvent switch from methanol to a nonpolar solvent after the separation by SFC. The later is described in the following section.

To achieve this solvent switch, an adapted setup is used, where a small $5 \mu \mathrm{L}$ injection loop is inserted into the $100 \mu \mathrm{L}$ sample loop, which is achieved by adding a third 6-port binary valve to the setup, as indicated in Figure 5.

This injection loop can be filled with a nonpolar solvent, e.g., toluene. After selecting the sample after SFC and switching in the injection loop with toluene, the plug consist of $3 \%$ methanol/ $\mathrm{CO}_{2}, 5 \mu \mathrm{L}$ toluene and $\gamma$-tocopherol. This plug is expanded to 48 bar at room temperature and the flow stopped once the plug reached the center of the stripline. The spectrum was recorded during $50 \mathrm{~min}$, averaging over 500 scans (Figure 6 , middle spectrum).

By injecting toluene and expanding the plug, the solvent is switched from methanol to toluene. The sample is separated in 


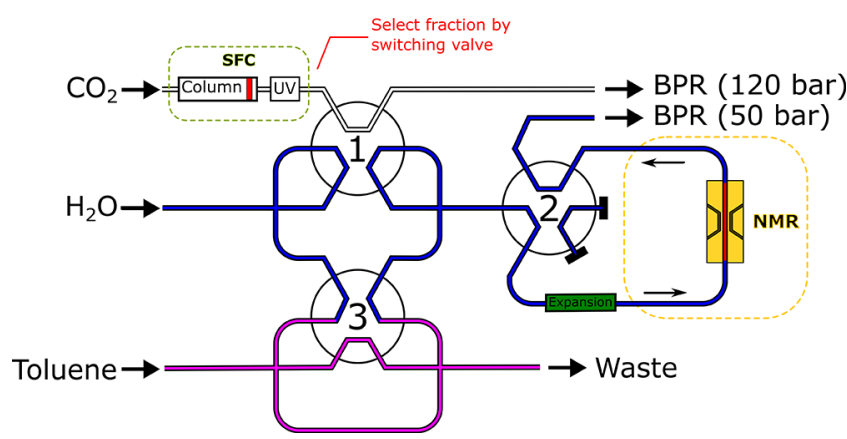

Figure 5. Valve switching system for selecting the desired sample from SFC (valve 1). A $5 \mu \mathrm{L}$ sample loop (pink) is added for injecting a small amount of toluene (valve 3 ). The flow can be stopped in the NMR by switching valve 2 .

a methanol $/ \mathrm{CO}_{2}$ mixture, but during the expansion the sample dissolves in toluene, while methanol partially mixes into the water flow at the trailing edge of the plug. In the middle spectrum in Figure 6, the aryl-H peak of tocopherol is visible, as was the case without solvent switch (upper spectrum, black). Although less toluene is now used ( $5 \mu \mathrm{L}$ vs $25 \mu \mathrm{L}$ ) to concentrate the sample, the toluene peak still has approximately the same intensity. This can be expected, since the toluene intensity is determined by the amount of toluene in the detection volume, not by the total toluene volume in the flow line. The aryl- $\mathrm{CH}_{3}$ peaks of the sample therefore still overlap with the toluene signal.

An additional advantage of the solvent switch procedure is that deuterated toluene (Deutero $\mathrm{GmbH}$, 99\% deuterated) can be used at limited cost, as the required volume is small, namely $5 \mu \mathrm{L}$. This avoids intense solvent peaks in the spectrum, as is shown in the bottom spectrum of Figure 6. Here the aryl-H as well as the two aryl- $\mathrm{CH}_{3}$ peaks are clearly resolved, confirming the correct selection of $\gamma$-tocopherol from the SFC chromatogram. Most of the water contamination that was previously present is removed as well, however a small methanol contamination is now observed, originating from the cosolvent that was used during chromatography. This does not interfere with the aryl- $\mathrm{H}$ and aryl- $\mathrm{CH}_{3}$ peaks, so the isomers can still be distinguished.

The experiment, including in-line expansion and solvent switch to $5 \mu \mathrm{L}$ toluene- $\mathrm{d}_{8}$, was repeated for $\alpha$ - and $\delta$ tocopherol (Figure 7). The concentrations of these isomers in

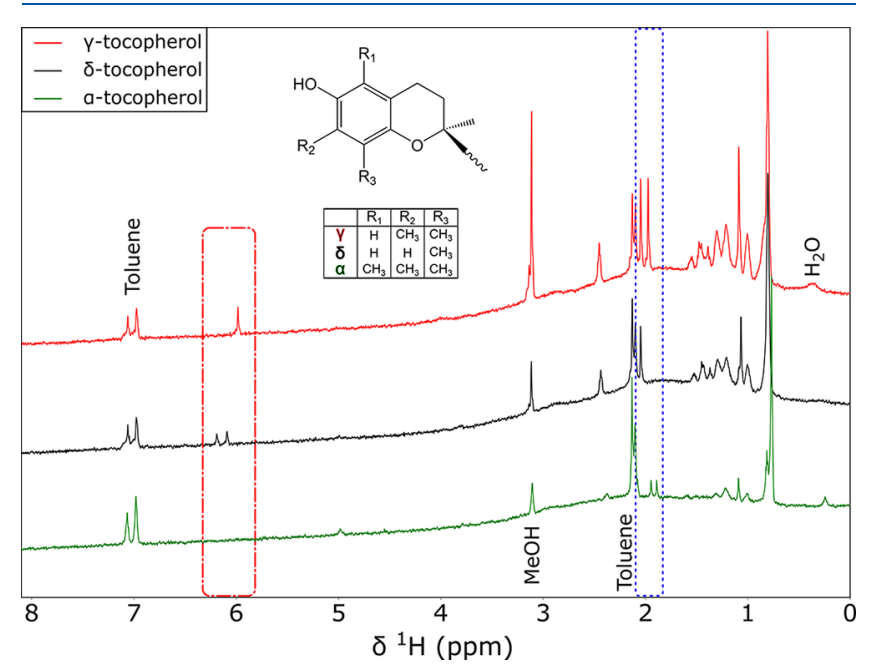

Figure 7. SFC $-{ }^{1} \mathrm{H}$ NMR spectrum of $\gamma$-tocopherol in methanol and toluene- $\mathrm{d}_{8}$ (red, same as Figure 6), of $\delta$-tocopherol in methanol and toluene- $\mathrm{d}_{8}$ (black) and of $\alpha$-tocopherol in methanol and toluene- $\mathrm{d}_{8}$ (green) obtained after selecting the correct isomer in SFC with 3\% methanol $/ \mathrm{CO}_{2}$ and expanding the plug to 50 bar at room temperature, in order to increase the concentration. 500 scans were averaged, taking $50 \mathrm{~min}$ per spectrum. The aryl- $\mathrm{H}$ (red) and aryl- $\mathrm{CH}_{3}$ (blue) peaks, which are needed to distinguish between the isomers of tocopherol, are clearly visible. One of the three methyl peaks of $\alpha$ tocopherol partially overlaps with the toluene peak, but can still be distinguished.
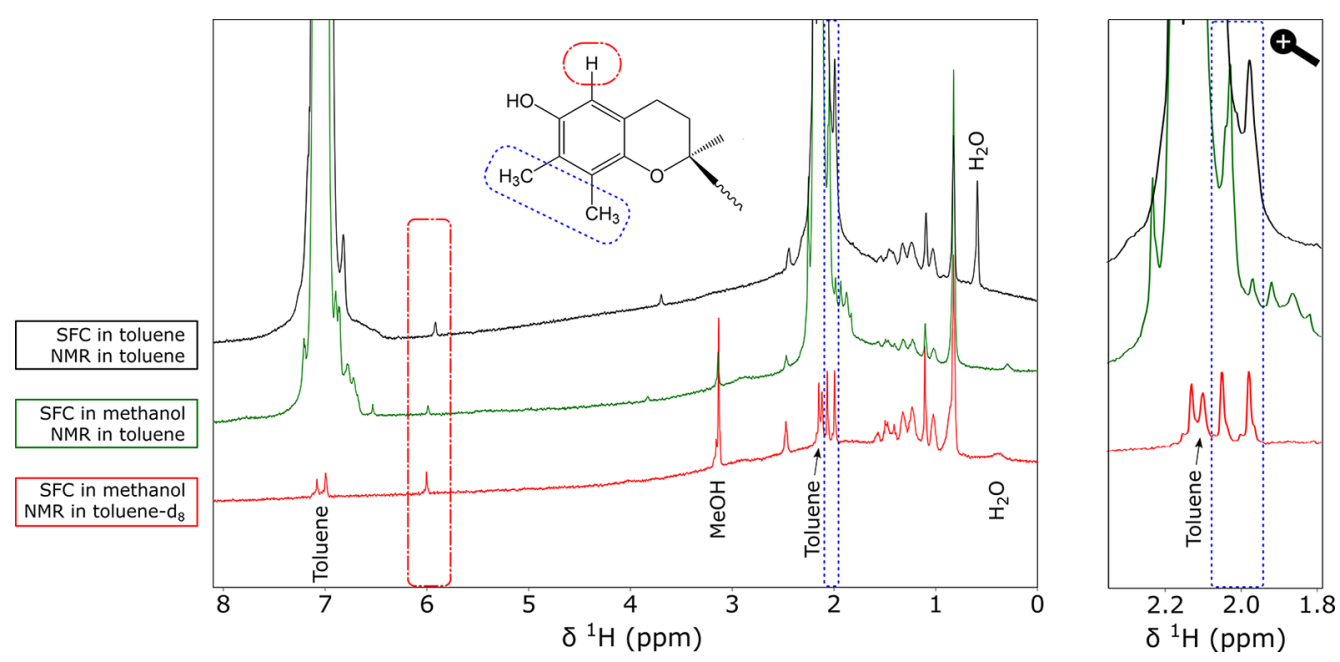

Figure 6. SFC- ${ }^{1} \mathrm{H}$ NMR spectrum of $\gamma$-tocopherol in methanol and toluene (green), obtained after selecting the correct isomer in SFC with $3 \%$ methanol $/ \mathrm{CO}_{2}$, switching solvents to toluene and expanding the plug to $50 \mathrm{bar}$ at room temperature, in order to increase the concentration. 500 scans were averaged and compared to 500 scans of the same experiment performed with 25\% toluene as SFC cosolvent (black, same as Figure 4). On the right the same spectra are shown as on the left, but zoomed in on the area around $2 \mathrm{ppm}$. The aryl- $\mathrm{H}$ peak (red), which is needed to distinguish between the isomers of tocopherol, is visible. The aryl- $\mathrm{CH}_{3}$ (blue) peaks overlap with the broad toluene peak. When using $5 \mu \mathrm{L}$ deuterated toluene for the solvent switch the bottom spectrum (red) is obtained. Here the aryl- $\mathrm{H}$ as well as the two aryl- $\mathrm{CH}_{3}$ peaks of $\gamma$-tocopherol are observed. 
the mixture were substantially lower than the $\gamma$-isomer $(66.7 \%$, determined by integrating the UV chromatogram), namely 9.7\% and $22.5 \%$ for $\alpha$ - and $\delta$-tocopherol, respectively. The $\beta$ isomer $(1.1 \%, 2.2 \mu \mathrm{g})$ was too low in concentration to be detected in 500 scans $(50 \mathrm{~min})$. The lowest amount of one single isomer that has to be injected on the column is therefore between $2.2 \mu \mathrm{g}(5.1 \mathrm{nmole})$ and $19 \mu \mathrm{g}(45.3 \mathrm{nmole})$ to be able to detect it in the SFC-NMR setup within $50 \mathrm{~min}$ of experiment time.

As shown, the concentration method was successfully extended to enable concentrating a nonpolar sample separated in SFC with $\mathrm{CO}_{2}$ and a polar cosolvent as the mobile phase, by switching to a nonpolar solvent in-line. This is applicable to other samples as well, as long as the sample dissolves better in the nonpolar NMR switching solvent than in the SFC cosolvent. The ability of switching solvents is an advantage since the best suitable solvent can be selected for SFC and NMR separately to match each sample. Care should be taken in the solvent selection to avoid sample precipitation in the SFC column or in the tubing toward the NMR spectrometer.

Quantifying the concentration step is possible by comparing the toluene peak integrals for the expanded (48 bar) and unexpanded (120 bar) toluene/ $\mathrm{CO}_{2}$ plug (SI S3). From these integrals it was calculated that due to the in-line expansion, approximately 10 times higher sample concentrations are observed, saving a factor of 100 in NMR experiment time. As stated before, the tocopherol mixture consisted of 9.7\% $\alpha$-, $1.1 \% \beta$-, $66.7 \% \gamma$-, and $22.5 \% \delta$-tocopherol. Selecting the highest abundant isomer, $\gamma$-tocopherol, and shuttling this compound directly to the NMR probe, without in-line concentration, would result in an isomer concentration of 0.2 $\mu \mathrm{g}(3.2 \mathrm{mM})$ in the $150 \mathrm{~nL}$ NMR detection volume of the stripline. On the basis of the LOD of the stripline chip mentioned in the introduction, this would be the lowest concentration that can be detected with the stripline probe within 500 scans ( $3 \mathrm{mM}$ in $50 \mathrm{~min}$ ). However, with the in-line concentration step described in this work, a 10 times higher sample concentration can be achieved in the stripline. Therefore, to end up with a concentration of $3 \mathrm{mM}$ in the stripline, 10 times less sample is needed. A concentration of 3 $\mathrm{mM}$ corresponds to $0.19 \mu \mathrm{g}$ tocopherol in $150 \mathrm{~nL}$, for which normally $0.13 \mathrm{mg}$ would need to be injected onto the SFC column, but with this concentration step only $13 \mu \mathrm{g}$ is needed. This coincides with the result that the $\alpha$-isomer can be detected $(9.7 \%, 19 \mu \mathrm{g}$ injected) after in-line concentration, but not the $\beta$-isomer (1.1\%, $2.2 \mu \mathrm{g}$ injected). As mentioned before, detecting the $\beta$-isomer would require concentrating the sample $\sim 57$ times, to $3 \mathrm{mM}$ in the stripline.

In the off-line experiment described by Tayler et al., ${ }^{22}$ the tocopherol isomers were collected 10 times after separation and redissolved in $0.5 \mu \mathrm{L}$ methanol- $\mathrm{d}_{4}$. The concentration in the stripline of $\beta$-tocopherol, the lowest abundant isomer, was therefore $3.2 \mathrm{mM}$, which is close to the detection limit for this stripline probe in 500 scans. In this case, without in-line concentration, the $\beta$-isomer concentration in the stripline is $0.053 \mathrm{mM}$. Therefore, to match the results of this off-line SFCNMR experiment, an in-line concentration step, concentrating the sample at least 61 times up to $3.2 \mathrm{mM}$, is needed. A higher concentration can be achieved by working at a higher temperature (SI S2), concentrating the sample 20-25 times instead of 10 times at room temperature, but this would still not be sufficient to detect the $\beta$-isomer.
As currently, the experimental times of the NMR analysis exceed the retention times in the SFC by far, storage loops are needed to perform a full analysis for a single injection of a (mass-limited) sample. To improve on this situation and to further bring down the LOD, the NMR sensitivity needs to increase. An easily perceived option is changing the detection volume of the stripline chip to better match the volume of the sample plugs. The sample is now concentrated in $5 \mu \mathrm{L}$ of toluene, of which only $150 \mathrm{~nL}$ can be detected with the current stripline probe. By making the detection volume larger, which is possible due to the scalability of the stripline, ${ }^{20}$ more sample is detected in one scan leading to a better signal-to-noise in the NMR spectrum. For example, increasing the volume with a factor 10 to $1.5 \mu \mathrm{L}$ leads to a 10 times higher signal, with the sensitivity of the stripline decreasing with its width by a factor of $\left.10^{(1 / 3)}(=2.15)\right)^{23}$ this would overall save a factor $\sim 50$ in experiment time. So the spectra which now took $50 \mathrm{~min}$ to acquire can then be acquired within $1 \mathrm{~min}$, making SFC-NMR viable for higher throughput analysis. For direct in flow detection of all fractions coming of the SFC columns further sensitivity enhancements are needed. This might be achieved by Overhauser dynamic nuclear polarization (DNP) for which $\mathrm{scCO}_{2}$ is a very efficient solvent. ${ }^{24}$ Developments along these lines are in progress.

\section{CONCLUSIONS}

A technique for concentrating samples in-line, which are diluted in $\mathrm{scCO}_{2}$ during chromatography was developed. This is achieved by controlled in-line expansion of the $\mathrm{scCO}_{2}$ to $\sim 50$ bar at room temperature. Even higher concentrations are achieved when expanding at a higher temperature of $\sim 90{ }^{\circ} \mathrm{C}$.

In-line SFC-NMR detection of $\alpha$-, $\gamma$-, and $\delta$-tocopherol is possible with this concentration step. Proof-of-principle experiments have been performed on a tocopherol mixture separated with SFC in toluene/ $/ \mathrm{sCO}_{2}$. Separation and concentration in methanol/ $\mathrm{scCO}_{2}$ is possible as well, but requires the addition of a small amount of toluene $(5 \mu \mathrm{L})$ after SFC separation to perform a solvent switch. The $\beta$-isomer concentration was too low to be detected, even after in-line concentration. Further research will focus on increasing the detection volume of the stripline to better match the sample volumes of $\sim 5 \mu \mathrm{L}$ which are obtained after in-line concentration. In this way more of the sample that is already present can be detected, possibly also $\beta$-tocopherol.

This in-line concentration technique is essential for coupling SFC and NMR in-line and could also be used in hyphenation of SFC with other detectors than NMR, such as MS. The hyphenation of SFC and NMR is interesting for studying supercritical behavior and mixing of solvents in supercritical fluids as well. Currently, the presented method is applicable for concentrating nonpolar samples in a polar or nonpolar cosolvent in $\mathrm{scCO}_{2}$. Further research is currently done to expand this method for concentrating and separating polar samples.

\section{ASSOCIATED CONTENT}

\section{Supporting Information}

The Supporting Information is available free of charge on the ACS Publications website at DOI: 10.1021/acs.analchem. 8 b02357.

S1: UV chromatogram of the separation of four tocopherol isomers. S2: Separation of toluene in 
$\mathrm{scCO}_{2}$ at elevated temperatures. S3: Quantification of concentration step. Calculation based on NMR spectra integration to express the efficiency of the in-line concentration step (PDF)

\section{AUTHOR INFORMATION}

\section{Corresponding Author}

*E-mail: A.Kentgens@nmr.ru.nl (A.P.M.K.).

\section{ORCID}

A. P. M. Kentgens: 0000-0001-5893-4488

\section{Notes}

The authors declare no competing financial interest.

\section{ACKNOWLEDGMENTS}

This research received funding from The Netherlands Organization for Scientific Research (NWO) in the framework of Technology Area COAST (053.21.115). The authors thank Julija Romanuka (Shell), Noud van den Borg (Waters), Peter Schoenmakers (UvA), and Ulrich Braumann (Bruker) for discussions and support and Hans Janssen (RU) for technical support.

\section{REFERENCES}

(1) Wilson, I. D.; Brinkman, U. A. T. Journal of Chromatography A 2003, 1000, 325-356.

(2) Silva-Elipe, M. V. Anal. Chim. Acta 2003, 497, 1-25.

(3) Albert, K.; Dachtler, M.; Glaser, T.; Händel, H.; Lacker, T.; Schlotterbeck, G.; Strohschein, S.; Tseng, L.-H.; Braumann, U. J. High Resolut. Chromatogr. 1999, 22, 135-143.

(4) Kesting, J. R.; Johansen, K. T.; Jaroszewski, J. W. Advances in Biomedical Spectroscopy 2011, 3, 413-434.

(5) Krucker, M.; Lienau, A.; Putzbach, K.; Grynbaum, M. D.; Schuler, P.; Albert, K. Anal. Chem. 2004, 76, 2623-2628.

(6) Lacey, M. E.; Tan, Z. J.; Webb, A. G.; Sweedler, J. V. Journal of Chromatography A 2001, 922, 139-149.

(7) Kühnle, M.; Kreidler, D.; Holtin, K.; Czesla, H.; Schuler, P.; Schaal, W.; Schurig, V.; Albert, K. Anal. Chem. 2008, 80, 5481-5486.

(8) Strohschein, S.; Pursch, M.; Lubda, D.; Albert, K. Anal. Chem. 1998, 70, 13-18.

(9) Seger, C.; Godejohann, M.; Tseng, L.-H.; Spraul, M.; Girtler, A.; Sturm, S.; Stuppner, H. Anal. Chem. 2005, 77, 878-885.

(10) Allen, L. A.; Glass, T. E.; Dorn, H. C. Anal. Chem. 1988, 60, 390-394.

(11) Braumann, U.; Händel, H.; Strohschein, S.; Spraul, M.; Krack, G.; Ecker, R.; Albert, K. Journal of Chromatography A 1997, 761, 336-340.

(12) Lindon, J. C.; Nicholson, J. K.; Wilson, I. D. Prog. Nucl. Magn. Reson. Spectrosc. 1996, 29, 1-49.

(13) Albert, K. Journal of Chromatography A 1997, 785, 65-83.

(14) Olson, D. L.; Peck, T. L.; Webb, A. G.; Magin, R. L.; Sweedler, J. V. Science 1995, 270, 1967-1970.

(15) Dechow, J.; Forchel, A.; Lanz, T.; Haase, A. Microelectron. Eng. 2000, 53, 517-519.

(16) Massin, C.; Vincent, F.; Homsy, A.; Ehrmann, K.; Boero, G.; Besse, P.-A.; Daridon, A.; Verpoorte, E.; De Rooij, N. F.; Popovic, R. S. J. Magn. Reson. 2003, 164, 242-255.

(17) Kentgens, A. P. M.; Bart, J.; Van Bentum, P. J. M.; Brinkmann, A.; Van Eck, E. R. H.; Gardeniers, J. G. E.; Janssen, J. W. G.; Knijn, P.; Vasa, S.; Verkuijlen, M. H. W. J. Chem. Phys. 2008, 128, 052202.

(18) Maguire, Y.; Chuang, I. L.; Zhang, S.; Gershenfeld, N. Proc. Natl. Acad. Sci. U. S. A. 2007, 104, 9198-9203.

(19) Webb, A. J. Pharm. Biomed. Anal. 2005, 38, 892-903 Quantitative NMR Spectroscopy.

(20) Van Bentum, P. J. M.; Janssen, J. W. G.; Kentgens, A. P. M.; Bart, J.; Gardeniers, J. G. E. J. Magn. Reson. 2007, 189, 104-113.
(21) Oosthoek-de Vries, A. J.; Bart, J.; Tiggelaar, R. M.; Janssen, J. W. G.; van Bentum, P. J. M.; Gardeniers, J. G. E.; Kentgens, A. P. M. Anal. Chem. 2017, 89, 2296-2303.

(22) Tayler, M. C. D.; van Meerten, S. G. J.; Kentgens, A. P. M.; van Bentum, P. J. M. Analyst 2015, 140, 6217-6221.

(23) Bart, J.; Janssen, J. W. G.; Van Bentum, P. J. M.; Kentgens, A. P. M.; Gardeniers, J. G. E. J. Magn. Reson. 2009, 201, 175-185.

(24) van Meerten, S. G. J.; Tayler, M. C. D.; Kentgens, A. P. M.; van Bentum, P. J. M. J. Magn. Reson. 2016, 267, 30-36. 